\title{
Planar Contour Tracking in the Presence of Pose and Model Errors by Kalman Filtering Techniques
}

\author{
Lyudmila Mihaylova* Herman Bruyninckx Joris De Schutter Ernesto Staffetti \\ Katholieke Universiteit Leuven, Department of Mechanical Engineering \\ Celestijnenlaan 300 B, B-3001 Heverlee, Belgium \\ Tel.: 00321632 25 33, E-mail: Lyudmila.Mihaylova@mech.kuleuven.ac.be
}

\begin{abstract}
The paper presents a solution to the problem of planar contour tracking with a force-controlled robot. The contour shape is unknown and is characterized at each time step by the curvature together with the orientation angle and arc length. The unknown contour curvature, continuously changing, is supposed to be within a preliminary given interval. An Interacting Multiple Model (IMM) filter is implemented to cope with the uncertainties. The interval of possible curvature values is discretized, i.e., a grid is formed and several Extended Kalman filters (EKFs) are run in parallel. The curvature estimate represents a fusion of the values from the grid with the IMM probabilities. The orientation angle estimate is also a fusion of the estimates, obtained from the separate Kalman filters with the mode probabilities. A single-model EKF is implemented to localize the unknown initial robot end-effector position over the contour. The performance of both algorithms is investigated and results, based on real data, are presented.
\end{abstract}

Key words - estimation, robotics, IMM filter, model and noise uncertainties, Kalman filter, force control

\section{INTRODUCTION}

Surface tracking along planar paths is required in several industrial applications such as cleaning, polishing and deburring of cast pieces. Often, these operations have to be executed by robots in the presence of uncertainties (e.g. for the piece position and orientation, shape deformations called burrs, calibration errors). Moreover, the robotic operations should be performed with a high speed and accuracy. Previous works devoted to problems of such kind are [4, 5, 2, 3, 7]. Different approaches have been developed, both modelbased $[4,5]$ and non-model-based [2, 3, 5], in which mathematical models are not present and look-up tables with data are used. A visual servoing-based deterministic approach has been proposed in $[2,3]$ where, with the help of a vision system, tracking and force control tasks are performed. The visual information is not only used to estimate the tool position around the contour, but also to predict approaching of sharp edges and corners.

This paper presents a solution for tracking contours with continuously varying curvature, based on the multiple-model approach, and, in particular, by the Interacting Multiple Model (IMM) estimator $[1,8]$. The case with known initial position over the contour is considered and the continuously varying curvature is replaced by discrete values within its uncertainty interval. The localization of the robot end-effector over the contour with known curvature and unknown initial position is performed by a single EKF.

\section{PROBLEM STATEMENT}

In many industrial applications a robot equipped with a tool is required to track planar contours moving with a given tangential velocity, keeping a specified orientation with respect to the normal of the contour, and applying a preset normal contact force. Encoders on the robot measure the end-effector's position in $x$ and $y$ directions.

In the present work the problem of tracking contours with an arbitrary form, like the object represented in Fig.1, is considered. To follow this contour the orientation angle $\theta$, the angle between the $x$ axis of the absolute (fixed) coordinate system and the tangent to the contour (Fig.2), is estimated. The normal and tangential directions are denoted by $n$ and $t$, respectively, and the applied force by $F_{n}$.

The following problems are considered here: i) identification of the contour geometry, i.e., computation of the contour curvature; ii) contour profile tracking (following) - the orientation angle $\theta_{k}$ has to be estimated at each time instant $k$, and also the curvature as a function of the arc length from the measured endeffector positions; iii) localization of the initial robot end-effector position along the contour.

* On leave from the Bulgarian Academy of Sciences 


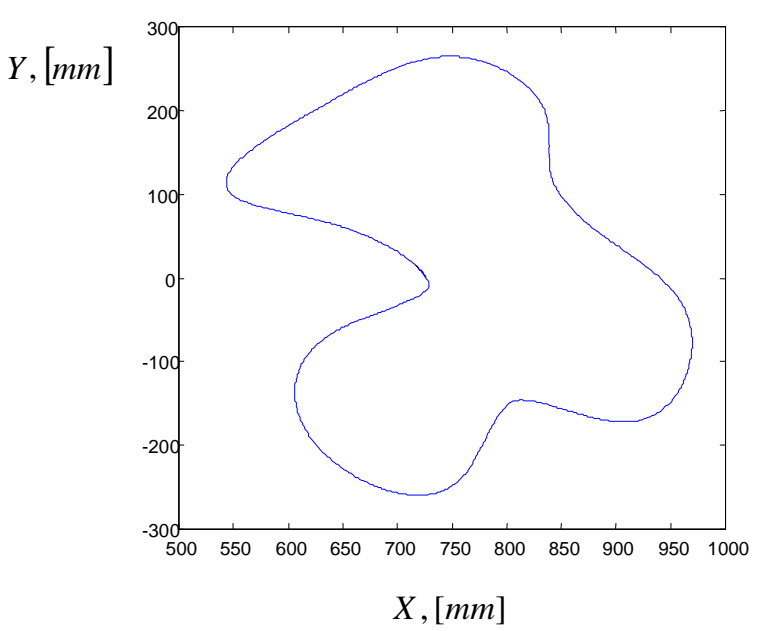

Fig.1. The considered planar contour

The velocities $v_{x, k}$ and $v_{y, k}$ of the tool in $x$ and $y$ directions with respect to the absolute coordinate system (Fig. 2) can be computed as follows

$$
v_{x, k}=\left(X_{k+1}-X_{k}\right) / T, v_{y, k}=\left(Y_{k+1}-Y_{k}\right) / T
$$

from the measured in two subsequent points positions $\left(X_{k}, Y_{k}\right)$ and $\left(X_{k+1}, Y_{k+1}\right)$, corrupted by noise. $T=1 / f$ denotes the sampling interval and $f$ the sampling frequency.

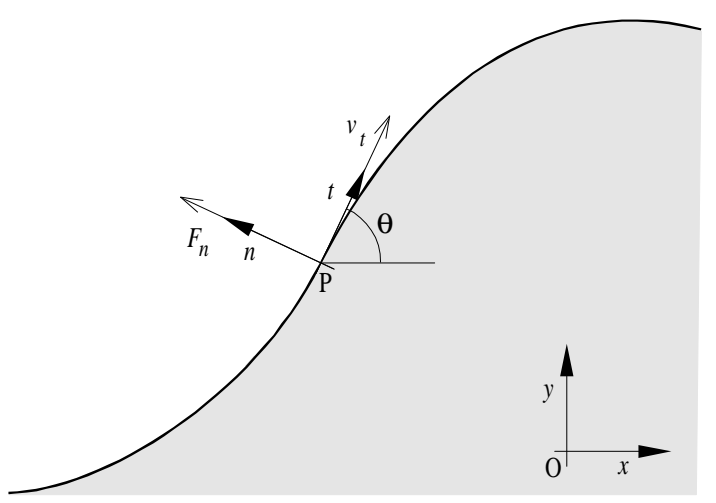

Fig. 2. The contour with the absolute (fixed) coordinate system $x O y$ and the moving coordinate system $n P t$, tangent to the contour

From the velocities of the tool the measured orientation angle $\theta_{k}$ can be determined at each time step as follows

$$
\theta_{k}=\arctan \frac{v_{x, k}}{v_{y, k}}
$$

and the measured curvature as

$$
k_{k}=-\frac{\omega_{k}}{v_{x, k}},
$$

where $\omega_{k}$ is the rotational velocity. Due to the presence of uncertainties as measurement errors, burrs (small contour deformations), the variables (1),(2) and (3) are inaccurate.

\section{IMM ALGORITHM FOR PLANAR CONTOUR TRACKING}

1.1 State and measurement models

Planar contour tracking can be considered as a hybrid stochastic estimation problem (with continuous and discrete uncertainties) and can be described by [1]

$$
\begin{aligned}
& x_{k+1}=f\left(x_{k}, m_{k}, \eta_{k}\right), \\
& z_{k}=g\left(x_{k}, \xi_{k}\right),
\end{aligned}
$$

where $x_{k}$ is the system state vector, $z_{k}$ is the measurement vector, $\eta_{k}$ and $\xi_{k}$ are respectively the process and measurement vectors, assumed zero mean white Gaussian noises, mutually uncorrelated, with covariances $Q$ and $R$. Functions $f($.$) and g($.$) are nonlinear$ in general. The unknown parameter $m_{k}$ corresponds here to the unknown contour curvature. It is supposed that the curvature change can be governed by a firstorder Markov chain with transition probabilities

$$
\pi_{i j, k}=P\left\{m_{j}(k+1) \mid m_{i}=m(k)\right\}, \quad i, j=1,2, \ldots, n_{f} .
$$

A state model of form (4) for the contour tracking problem (Fig. 1) can be derived from the fact that the continuously varying curvature $k$ represents the derivative of the orientation angle $\theta$ with respect to the arc length $s$ [6]

$$
k=\frac{d \theta}{d s} .
$$

Equation (6), written in a discrete form with respect to the orientation angle is

$$
\theta_{k+1}=\theta_{k}+k_{k} \Delta s_{k},
$$

and taking into account that the arc length is the integral of the constant tangential velocity $v_{t}$ of the robot end-effector, it follows

$$
\theta_{k+1}=\theta_{k}+k_{k} v_{t} T \text {. }
$$

The unknown curvature of the contour is supposed to vary in a preset interval: $k_{k} \in\left[k_{\min }, k_{\max }\right]$. It is assumed that the signed curvature is negative on locally convex curve segments and positive on locally concave curve segments $[4, \mathrm{p} .35]$. The estimated state variable $x_{k}$ is the orientation angle $\theta$, i.e. $x_{k}=\theta_{k}$. Since $k$ is unknown, multiple models for $\theta_{k}$ are constructed with 
different curvature values $k_{i}$ from the uncertainty interval. Then the state equation has the form:

$$
x_{i, k+1}=x_{i, k}+k_{i} v_{t} T+\eta_{k} .
$$

The index $i$ denotes different models depending on the curvature values. The process noise reflects discretization and model errors.

\subsection{EKFs for Unknown Contour Tracking}

The measured data are the positions $X_{k}$ and $Y_{k}$ of the robot end-effector, on the basis of which the velocities $v_{x, k}$ and $v_{y, k}$ are computed by (1). The velocity $v_{y, k}$, corrupted by the noise $\xi_{k}$

$$
z_{k}=v_{y, k}+\xi_{k},
$$

is used as measurement sequence $\left\{z_{k}\right\}$ for the filtering. The measurement function $z_{k}=g\left(x_{k}\right)$ can be expressed in the following way ${ }^{1}$

$$
g\left(x_{k}\right)=v_{y, k}=v_{x, k} \tan \left(x_{k}\right) .
$$

With the state equations (8) and the measurement equation (9), several Extended Kalman filters are synthesized :

$$
\begin{aligned}
& \hat{x}_{i, k+1 / k+1}=\hat{x}_{i, k+1 / k}+K_{i, k+1} v_{i, k+1}, \\
& \hat{x}_{i, k+1 / k}=\hat{x}_{i, k / k}+k_{i} v_{t} T, \\
& v_{i, k+1}=z_{k+1}-g\left(\hat{x}_{i, k+1 / k}\right), \\
& P_{i, k+1 / k}=P_{i, k / k}+Q_{k}, \\
& K_{i, k+1}=P_{i, k+1 / k} g_{x_{i}, k+1} S_{i, k+1}^{-1}, \\
& P_{i, k+1 / k+1}=P_{i, k+1 / k}-K_{i, k+1} S_{i, k+1} K_{i, k+1}, \\
& S_{i, k+1}=g_{x_{i}, k+1} P_{i, k+1 / k+1} g_{x_{i}, k+1}+R_{k+1},
\end{aligned}
$$

where $\hat{x}_{i, k / k}$ is the filtered state estimate; $\hat{x}_{i, k / k-1}$ is the one-step predicted state estimate; $P_{k / k}$ is the state estimation covariance; $v_{i, k}$ is the innovation with covariance $S_{i, k}$;

$$
g_{x_{i}, k+1}=\partial g_{k}\left(\hat{x}_{i, k+1 / k}\right) / \partial \hat{x}_{i, k+1 / k}
$$

is the measurement Jacobian

$$
g_{x_{i}, k+1}=\frac{v_{x, k+1}}{\cos ^{2}\left(\hat{x}_{i, k+1 / k}\right)} .
$$

\footnotetext{
1 Equation (9) is applied when $-\pi / 4 \leq x_{k}=\theta_{k} \leq \pi / 4$ or $-3 \pi / 4 \leq x_{k}=\theta_{k} \leq 5 \pi / 4$. Outside these intervals it is not well conditioned numerically, and it is replaced by $g\left(x_{k}\right)=v_{x, k}=v_{y, k} \cot \left(x_{k}\right)$
}

Within the framework of the IMM approach [8], the overall estimate (here the orientation angle estimate) represents the probabilistically weighted sum of the estimates from the filters working in parallel, i.e.

$$
\hat{x}_{k / k}=\sum_{i=1}^{n_{f}} \hat{x}_{i, k / k} \mu_{i, k},
$$

where $n_{f}$ is the number of filters and $\mu_{i, k}$ are the IMM mode probabilities. The mode probabilities are an indicator at each moment what model describes the state the most adequately.

The contour curvature can also be evaluated as a probabilistically weighted sum of preset curvature values from the uncertainty interval with the mode probabilities $\mu_{i, k}$

$$
\hat{k}_{k}=\sum_{i=1}^{n_{f}} k_{i} \mu_{i, k} \text {. }
$$

The arc length estimate $\hat{s}_{k}$ is computed as follows

$$
\hat{s}_{k}=s_{0}+\sum_{j=1}^{k} \Delta \hat{s}_{j},
$$

where

$$
\Delta \hat{s}_{k}=\sqrt{\left(X_{k+1}-X_{k}\right)^{2}+\left(Y_{k+1}-Y_{k}\right)^{2}},
$$

and $s_{0}$ is the initial arc length shift, characterizing the initial position over the contour.

\section{EKF FOR LOCALIZATION OF THE ROBOT END-EFFEC- TOR POSITION OVER A CONTOUR WITH KNOWN SHAPE}

After the estimation of the curvature of the contour, its shape is yet known. It is also important in some robotic applications to localize the starting unknown endeffector position $s_{0}$ over the contour. In this case the estimated state represents the arc length shift, i.e. $x=s_{0}$. Theoretically $x$ remains constant.

The state equation is of the form

$$
x_{k+1}=x_{k},
$$

i.e. no noise is present and hence the system covariance $Q=0$.

The measurements are the noisy curvature values, provided by (3). The measurement function

$$
z_{k}=h\left(x_{k}, \zeta_{k}\right)=k\left(\hat{s}_{k}\right),
$$

is derived from the estimated curvature profile (Fig.10). $\zeta_{k}$ is the measurement noise with a covariance $R_{\zeta}$. The analytical derivative $h_{x}=\partial k / \partial \hat{s}$ on segments with 
constant curvature can not be found and it is replaced by the curvature computed with finite differences

$$
h_{x, k+1}=\left(\hat{\kappa}_{k+1}-\hat{k}_{k}\right) / \Delta \hat{s}_{k} \text {. }
$$

The filter equations are

$$
\begin{aligned}
& \hat{x}_{k+1}=\hat{x}_{k}+K_{k+1} v_{k+1}, \\
& P_{k+1 / k}=\lambda P_{k / k}, \\
& K_{k+1}=P_{k+1} h_{x, k+1} P_{k+1} S_{k+1}^{-1}, \\
& P_{k+1 / k+1}=P_{k+1 / k}-K_{k+1} S_{k+1} K_{k+1}, \\
& S_{k+1}=h_{x, k+1} P_{k+1 / k+1} h_{x, k+1}+R_{\zeta, k+1},
\end{aligned}
$$

where $\lambda$ is the fudge factor improving the filter performance, the innovation $v_{k}$ represents the difference between the measured noisy curvature (3) and the curvature identified by (17)

$$
v_{k+1}=k_{k+1}-\hat{k}_{k+1} \text {. }
$$

\section{PERFORMANCE ANALYSIS}

The following measures of performance are used: the estimated curvature (representing a fusion between the values from the grid weighted by the mode probabilities), the normalized innovation squared (NIS) (characterising the estimate consistency) [1], the mode probabilities, the averaged overall state estimate, compared to the noise-corrupted (measured) angle $\theta_{k}$.

The normalized innovation squared $\varepsilon_{v, k}$ is computed from the "averaged" innovation (as a probabilistically weighted sum of the innovations from the EKFs) and its covariance $S_{k}$ as follows $\varepsilon_{v, k}=v_{k} S_{k}^{-1} v_{k}$.

The results presented are based on processing of real experimental data for one tour around the contour. The interval for the curvature values is: $k_{k} \in[-0.04,0.15]$ $\mathrm{mm}^{-1}$, determined from the preliminary available information.

The designed IMM filter is implemented with a set of six values for the curvature, namely

$$
K^{6}=\{-0.04,-0.01,0,0.03,0.05,0.135,0.15\} \mathrm{mm}^{-1}
$$

chosen to cover well the uncertainty interval. The measurement covariance $R$ is experimentally determined, from the information of the sensor errors and it is $R=0.001(\mathrm{~mm} / \mathrm{s})^{2}$. The system noise covariance is $Q=0.005 \mathrm{rad}^{2}$. It reflects the expected error from the substitution of the continuously varying curvature by a set of discrete values. The experimental data are received with a sampling frequency $f=10[\mathrm{~Hz}]$ and the tangential velocity is constant, known to be $v_{t}=20[\mathrm{~mm} / \mathrm{s}]$.
The IMM estimator is run with the following initial transition probability matrix and the mode probability vector

$$
\begin{gathered}
\pi_{0}=\left[\begin{array}{lllllll}
0.940 & 0.012 & 0.012 & 0.012 & 0.012 & 0.012 \\
0.012 & 0.940 & 0.012 & 0.012 & 0.012 & 0.012 \\
0.012 & 0.012 & 0.940 & 0.012 & 0.012 & 0.012 \\
0.012 & 0.012 & 0.012 & 0.940 & 0.012 & 0.012 \\
0.012 & 0.012 & 0.012 & 0.012 & 0.940 & 0.012 \\
0.012 & 0.012 & 0.012 & 0.012 & 0.012 & 0.940
\end{array}\right], \\
\mu_{0}=\left[\begin{array}{llllll}
1 / 6 & 1 / 6 & 1 / 6 & 1 / 6 & 1 / 6 & 1 / 6
\end{array}\right]^{T},
\end{gathered}
$$

the initial state estimates and covariances are $x_{i, 0}=0$, $P_{i, 0}=10^{5}$. The initial mode probabilities are chosen equal, which corresponds to the lack of information about the contour curvature.

The NIS test and the averaged innovation process characterize the transitions from parts with different curvature. The plots of the estimated and measured curvature (Fig. 3), the estimated orientation angle (Fig. $4)$, the error between the estimated and measured angle (Fig.5), the normalized innovation squared $\varepsilon_{v, k}$ (Fig.6), the IMM mode probabilities (Figs. 7-9), and the estimated curvature with respect to the arc length (Fig. 10) are given. As a result of filtering the estimated curvature is smoothed and at each moment the contour is tracked. The mode probabilities provide information about switching between the different curvature values.

The performance evaluation results of the EKF (19)-24) for the starting point localization are given in Fig. 11 and Fig.12. The measurement noise covariance is $R_{\zeta}=1.6 \mathrm{~mm}^{-2}$ and the fudge factor $\lambda=1.01$. The real robot end-effector initial arc length shift is $s_{0}=20 \mathrm{~mm}$. The estimation accuracy and the rate of convergence of this EKF depend on the accuracy of the estimates for the curvature and the arc length shift. The EKF (19)-24)

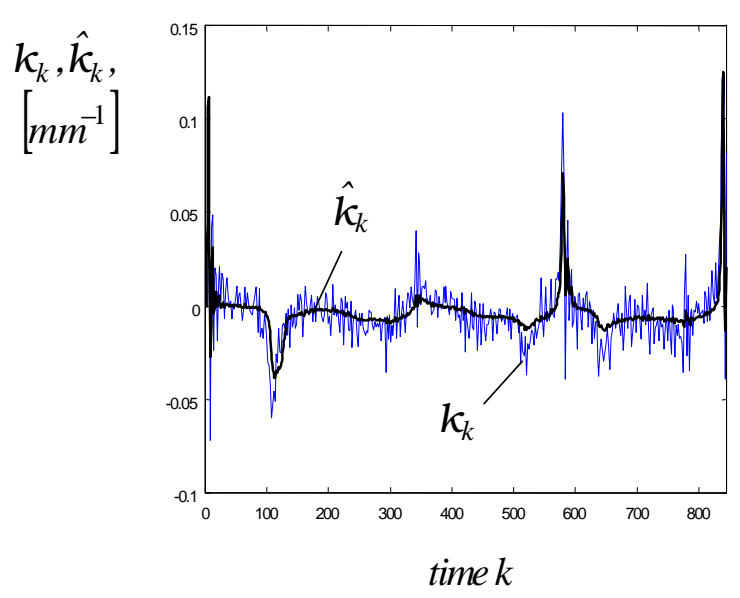

Fig. 3. Estimated $\hat{k}_{k}$ and measured $k_{k}$ curvature 
$\hat{\theta}_{k},[\mathrm{rad}]$

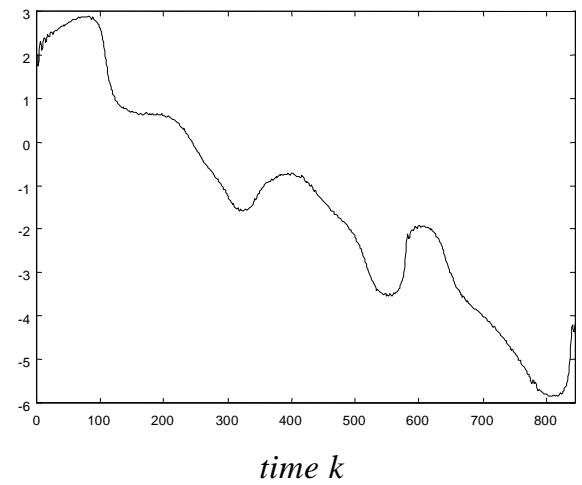

Fig. 4. Estimated orientation angle $\hat{\theta}_{k}$

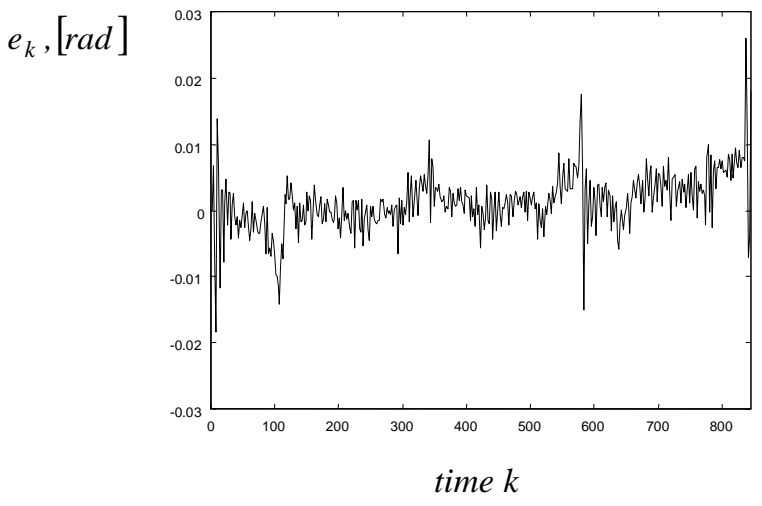

Fig. 5. Error $e_{k}=\theta_{k}-\theta_{k}$ between the measured and estimated orientation angle

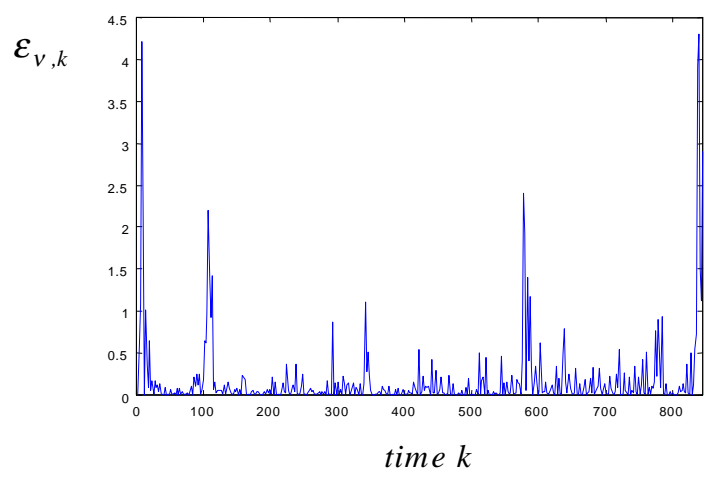

Fig. 6. Normalized Innovation Squared $\varepsilon_{v, k}$

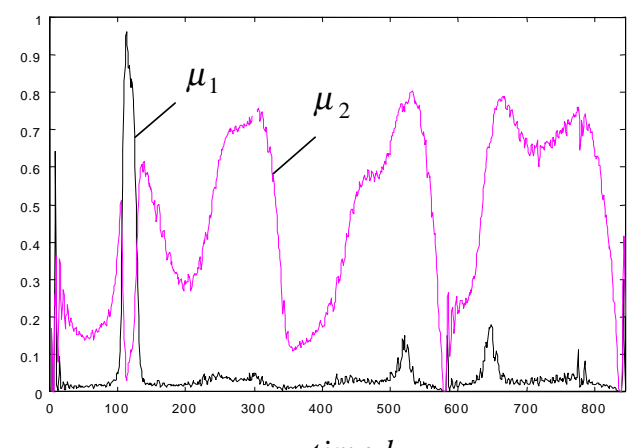

time $k$

Fig. 7. IMM mode probabilities $\mu_{1}$ and $\mu_{2}$

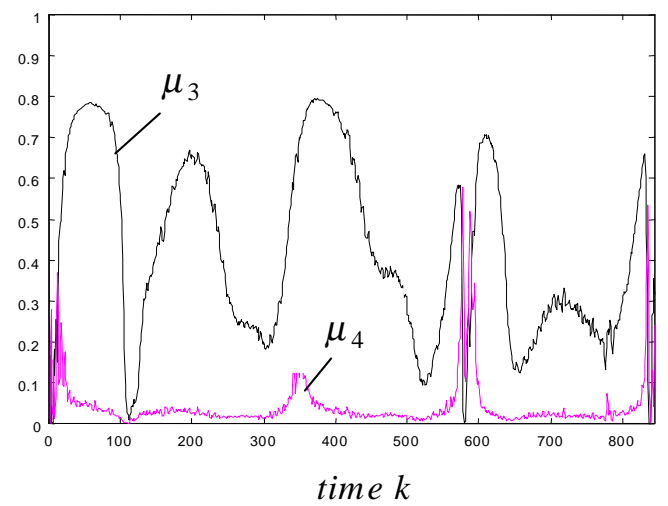

Fig. 8. IMM mode probabilities $\mu_{3}$ and $\mu_{4}$

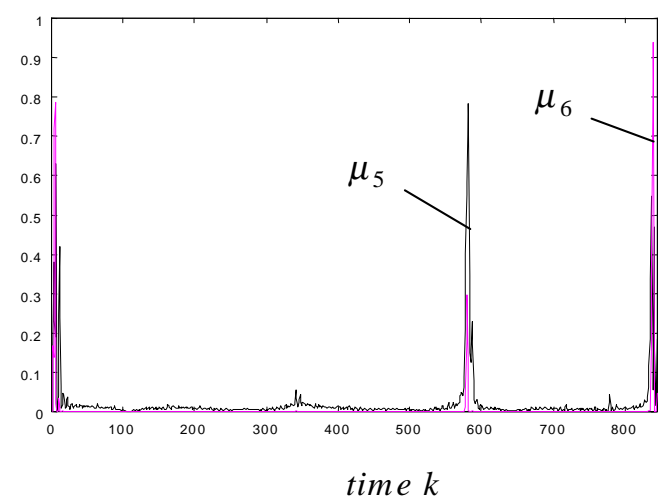

Fig. 9. IMM mode probabilities $\mu_{5}$ and $\mu_{6}$

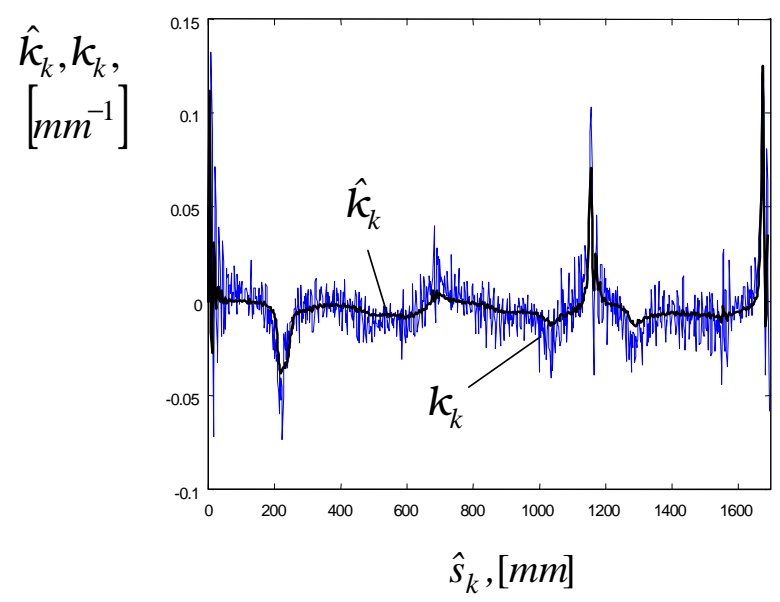

Fig. 10. Estimated curvature $\hat{k}_{k}$ with respect to the estimated arc length $\hat{s}_{k}$

is working with the curvature estimated by the IMM algorithm. Because the provided by the IMM curvature estimate is accurate, respectively the computed estimate for the initial position over the contour is accurate. The EKF consistency, characterized by the NIS test is also better (Fig. 12) than the NIS test of the presented in [4] 
similar EKF. In [4] the identified curvature values are taken from a CAD model of the undeformed contour model, whereas here the results obtained by the IMM algorithm are used. As obvious from Fig. 11, a quick convergence toward the true value is observed.

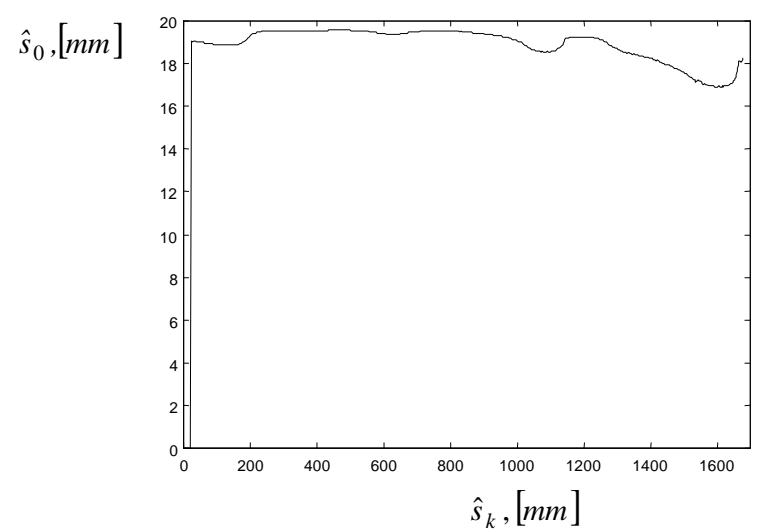

Fig. 11 Estimated arc length shift with respect to the arc length estimate

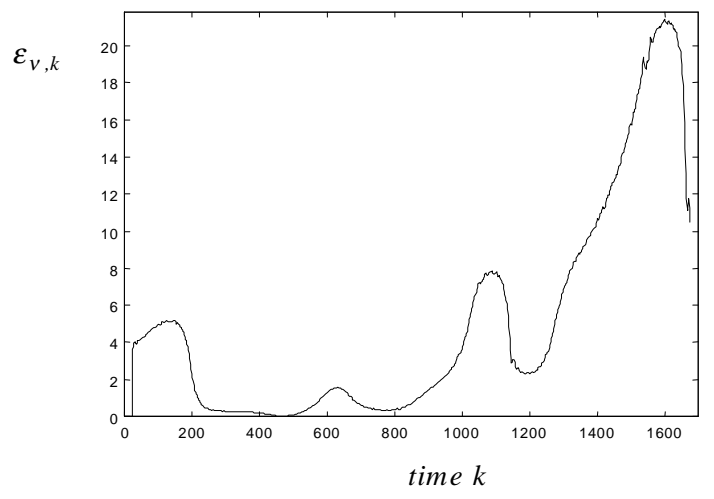

Fig. 12 Normalized Innovation Squared $\varepsilon_{v, k}$

\section{CONCLUSIONS}

In this work the problem of planar contour tracking in the presence of pose and model errors has been considered as a hybrid stochastic estimation problem.

An IMM filter has been implemented and its performance evaluated. The inherent characteristic of the IMM filter gives the possibility to estimate on-line the contour curvature and the orientation angle on the basis of a set of values for the curvature taken from a preset interval. So, both contour shape identification and tracking are performed. The IMM mode probabilities provide information about the position of the tool over different parts of the contour.
The multiple model approach can be extended to other similar problems, such as tracking of curved surfaces, if suitable mathematical models are provided.

The problem of localization of the tool over a planar contour (estimation of the initial position) has also been considered. A single EKF is synthesized. The performance of the implemented algorithms is characterized by the normalized innovation squared test and the estimated variables. Results from experiments with real data are presented.

\section{AcKNowledgments}

H. Bruyninckx is a Postdoctoral Fellow of the Fund for Scientific Research-Flanders (F.W.O) in Belgium. Financial support by the Belgian Programme on InterUniversity Attraction Poles initiated by the Belgian State-Prime Minister's Office-Science Policy Programme (IUAP), the F.W.O. under grant G.0295.96N, K.U.Leuven's Concerted Research Action GOA/99/04 is gratefully acknowledged.

\section{REFERENCES}

[1] Bar-Shalom, Y., and X.R. Li, Estimation and Tracking: Principles, Techniques and Software, Artech House, 1993.

[2] Baeten, J., W. Verdonck, H. Bruyninckx, and J. De Schutter, Combining Force Control and Visual Servoing for Planar Contour Following, Machine Intelligence \& Robotic Control, Vol. 2, No. 2, pp. 3-9, 2000.

[3] Baeten, J., H. Bruyninckx, and J. De Schutter, Combining Eye-in-hand Visual Servoing and Force Control in Robotic Tasks Using the Task Frame, In Proc. of 1999 IEEE International Conf. on Multisensor Fusion and Integration for Intelligent Systems, Taipei, Taiwan, Aug. 1999, pp. 141-146.

[4] Demey S., H. Bruyninckx, and J. Schutter, ModelBased Planar Contour Following in the Presence of Pose and Model Errors, International Journal of Robotics Research, Vol. 16, No. 6, December 1997, pp.840-858.

[5] Demey, S., Shape Identification an Shape Matching for Compliant Motion Based on Invariant Differential Shale Descriptions, $\mathrm{PhD}$ thesis, Katholieke Universiteit Leuven, 1996.

[6] Do Carmo, M., Differencial Geometry of Curves and Surfaces, N.J., Prentice Hall, 1976.

[7] Gorinevsky, D., A. Formalsky, A. Schneider, Force Control of Robotics Systems, CRC Press, New York, 1997.

[8] Li, X. R. Hybrid Estimation Techniques. Control and Dynamic Systems. (Ed. C. T. Leondes), Vol. 76, pp. 213-287, Academic Press, Inc., 1996. 